\title{
Influence of heart rate on coronary calcium scores: a multi- manufacturer phantom study
}

\author{
N. R. van der Werf ${ }^{1,2,3,4} \cdot$ M. J. Willemink ${ }^{1} \cdot$ T. P. Willems ${ }^{2} \cdot$ R. Vliegenthart ${ }^{2} \cdot$ M. J. W. Greuter ${ }^{2} \cdot$ T. Leiner $^{1}$
}

Received: 30 October 2017 / Accepted: 19 December 2017 / Published online: 28 December 2017

(c) The Author(s) 2017. This article is an open access publication

\begin{abstract}
To evaluate the influence of heart rate on coronary calcium scores (CCS) using a dynamic phantom on four high-end computed tomography (CT) systems from different manufacturers. Artificial coronary arteries were moved in an anthropomorphic chest phantom at linear velocities, corresponding to $<60,60-75$ and $>75$ beats per minute (bpm). Data was acquired with routinely used clinical protocols for CCS on four high-end CT systems (CT1-CT4). CCS, quantified as Agatston and mass scores were compared to reference scores at $<60 \mathrm{bpm}$. Influence of heart rate was assessed for each system with the cardiac motion susceptibility (CMS) Index. At increased heart rates (> $75 \mathrm{bpm})$, Agatston scores of the low mass calcification were similar to the reference score, while Agatston scores of the medium and high mass calcification increased significantly up to $50 \%$ for all CT systems. Threefold CMS increases at $>75 \mathrm{bpm}$ in comparison with $<60 \mathrm{bpm}$ were shown. For medium and high mass calcifications, significant differences in CMS between CT systems were found. Heart rate substantially influences CCS for high-end CT systems of four major manufacturers, but CT systems differ in motion susceptibility. Follow-up CCS CT scans should be acquired on the same CT system and protocol, and preferably with comparable heart rates.
\end{abstract}

Keywords Computed tomography $\cdot$ Coronary calcium $\cdot$ Agatston score $\cdot$ Mass score $\cdot$ Heart rate

$\begin{array}{ll}\text { Abbreviations } \\ \text { BPM } & \text { Beats per minute } \\ \text { CCS } & \text { Coronary calcium score } \\ \text { CMS } & \text { Cardiac Motion Index } \\ \text { CT } & \text { Computed tomography } \\ \text { FBP } & \text { Filtered back projection } \\ \text { HA } & \text { Hydroxyapatite } \\ \text { HU } & \text { Hounsfield units }\end{array}$

N. R. van der Werf

n.r.vanderwerf@umcutrecht.nl; n.r.vanderwerf@asz.nl

M. J. Willemink

m.j.willemink@gmail.com

T. P. Willems

t.p.willems@umcg.nl

R. Vliegenthart

r.vliegenthart@umcg.nl

M. J. W. Greuter

m.j.w.greuter@umcg.nl

T. Leiner

t.leiner@umcutrecht.nl

\section{Introduction}

Despite advances in treatment, atherosclerotic cardiovascular disease remains the main cause of mortality worldwide [1]. For the determination of the prognosis of future cardiovascular disease, risk categories based on the amount of coronary calcium are increasingly used [2]. Coronary calcium can be quantified with computed tomography (CT) as coronary calcium scores (CCS), including Agatston scores, mass scores and volume scores [3]. Correct and reproducible CCS are essential considering their use in the guidelines [1,

1 Department of Radiology, University Medical Center Utrecht, Heidelberglaan 100, 3584 CX Utrecht, The Netherlands

2 Department of Radiology, Center for Medical Imaging, University Medical Center Groningen, University of Groningen, Hanzeplein 1, 9713 GZ Groningen, The Netherlands

3 Department of Clinical Physics, Albert Schweitzer Hospital, Albert Schweitzerplaats 25, 3318 AT Dordrecht, The Netherlands

4 Department of Radiology, University Medical Center Utrecht, E01.132, PO Box 85500, 3508 GA Utrecht, The Netherlands 
4]. If risk-based treatment decision is uncertain after quantitative risk assessment, the 2013 guidelines recommend repeated CCS measurements. Also, CCS progression in repeated CCS measurements has recently been associated with heart failure [5].

Several studies have demonstrated the dependence of CCS on motion [6-9]. Motion artifacts can result in incorrect Agatston scores, which may thereby lead to incorrect risk classification. Depending on heart rate and anatomical location, coronary arteries move at velocities of $10-30 \mathrm{~mm} / \mathrm{s}$ during the acquisition phase, whereas coronary arteries are often erroneously assumed to be stationary during CT acquisition [10-12].

Previously published studies on the influence of heart rate on CCS have only focused on CT systems from a single manufacturer [9, 13, 14]. Recently, substantial differences in CCS between new generation CT systems were demonstrated. Willemink et al. found differences in Agatston scores between CT systems of up to $43.9 \%$ for static calcifications [15]. However, the effect of heart rate on CCS for different state-of-the-art CT systems remains unknown. The aim of the current study was therefore to determine the influence of heart rate on CCS for the high-end CT systems from four major manufacturers at routinely used clinical protocols with a dynamic phantom.

\section{Materials and methods}

\section{Phantom}

A computer-controlled lever (QRM-Sim2D, QRM, Möhrendorf, Germany) moved an artificial coronary artery in a water container in the center of an anthropomorphic chest phantom (QRM-Chest, QRM, Möhrendorf, Germany) (Fig. 1). The chest phantom consisted of artificial lungs, a spine insert and a shell of tissue equivalent material. An extension ring (QRM-Extension Ring, QRM, Möhrendorf, Germany), made of fat equivalent material, was used to increase the size of the phantom to $400 \times 300 \mathrm{~mm}$, in order to mimic an average sized patient. The artificial artery was moved at constant linear velocities of $0,10,20$ and $30 \mathrm{~mm} / \mathrm{s}$ in the horizontal plane, perpendicular to the scan direction. Artificial calcified coronary arteries were used, which contained cylindrical calcifications of calcium hydroxyapatite (HA) (Fig. 2). The dimensions of the calcifications were $5.0 \pm 0.1 \mathrm{~mm}$ in diameter and $10.0 \pm 0.1 \mathrm{~mm}$ in length. Three calcification densities were used: $196 \pm 3,408 \pm 2$ and $800 \pm 2 \mathrm{mg} \mathrm{HA} / \mathrm{cm}^{3}$. These densities were categorized as low $(38.5 \pm 1.7 \mathrm{mg})$, medium $(80.1 \pm 3.3 \mathrm{mg})$ and high $(157.1 \pm 6.5 \mathrm{mg})$, corresponding to mild, moderate and severe calcified coronary plaque burden, respectively.

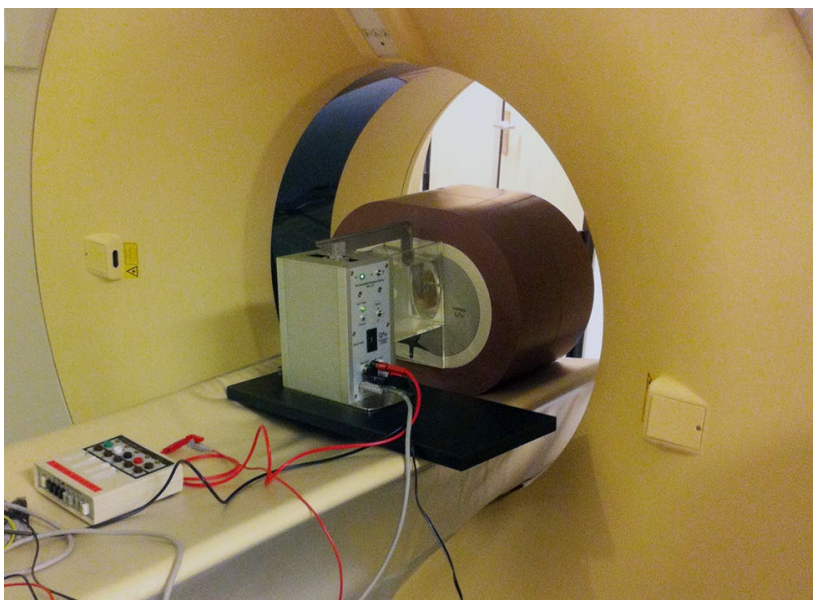

Fig. 1 Anthropomorphic chest phantom with extension ring and motion controller (QRM, Moehrendorf, Germany). An artificial coronary artery moved in the horizontal plane inside the water tank in the center of the chest phantom

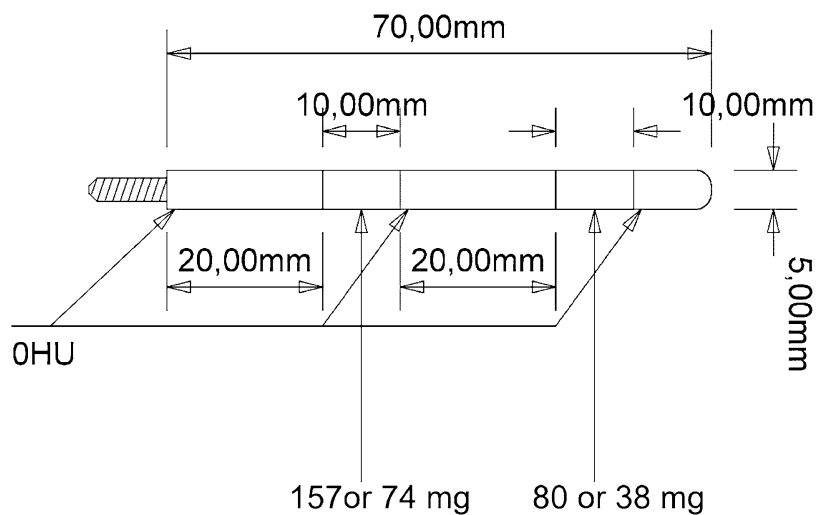

Fig. 2 The cylindrical artificial coronary artery contained two calcified inserts with a diameter of $5.0 \pm 0.1 \mathrm{~mm}$ and a length of $10.0 \pm 0.1 \mathrm{~mm}$ as indicated

\section{Image acquisition and evaluation}

In order to assess the influence of heart rate on CCS in a clinical setting, routinely used clinical CT CCS protocols were used (Table 1). The protocols were equal to the manufacturer recommended protocol if available or were adapted from the factory settings based on recommendations by the specific manufacturer consultants. Four high-end CT systems from the main four CT manufacturers were used (CT1-CT4): Discovery CT 750 HD (GE Healthcare, Waukesha, WI, USA), Brilliance iCT (Philips Healthcare, Best, The Netherlands), Somatom Definition Flash (Siemens Healthcare, Forchheim, Germany) and Aquilion One (Toshiba Medical Systems, Otawara, Japan), respectively. 
Table 1 Acquisition and reconstruction parameters used on CT system CT1-CT4

\begin{tabular}{lllll}
\hline CT system & CT1 & CT2 & CT3 & CT4 \\
\hline Tube voltage (kV) & 120 & 120 & 120 & 120 \\
Tube current per rotation (mA) & 175 & 50 & 80 & 80 \\
Collimation (mm) & $64 \times 0.625$ & $128 \times 0.625$ & $128 \times 0.6$ & $320 \times 0.5$ \\
Rotation time (ms) & 350 & 270 & 280 & 350 \\
Temporal resolution ${ }^{\mathrm{a}}(\mathrm{ms})$ & 175 & 135 & 75 & 175 \\
Slice thickness $(\mathrm{mm})$ & 2.5 & 3.0 & 3.0 & 3.0 \\
Increment $(\mathrm{mm})$ & 2.5 & 3.0 & 3.0 & 3.0 \\
Kernel & Standard & XCA & B35f & FC12 \\
Calcium scoring software & Smartscore 4.0 & Heartbeat-CS & Syngo.via & Vitrea FX 6.5.0 \\
\hline
\end{tabular}

${ }^{\mathrm{a}}$ As defined in the isocenter

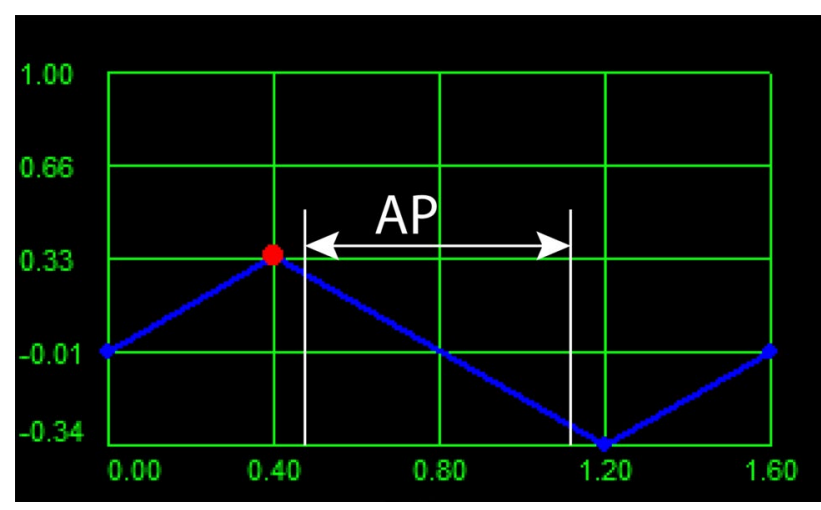

Fig. 3 Example profile for $30 \mathrm{~mm} / \mathrm{s}$ (>75 bpm) movement of the Sim2D motion controller. The red dot resembles the ECG triggering point, after which acquisition was started. For all CT systems, acquisition was in the acquisition phase (AP), as indicated in the figure. Therefore, all acquisitions were during constant motion of the motion controller

For each velocity the phantom was scanned five times, with a small random translation $(2 \mathrm{~mm})$ and rotation $\left(2^{\circ}\right)$ between the scans by repositioning of the phantom. An electrocardiographic trigger output of the motion controller was used for electrocardiographic triggering of the CT systems to ensure that data was acquired only during linear motion of the phantom (Fig. 3). Data were reconstructed with filtered back projection (FBP) on each CT system using the kernels listed in Table 1. CCS was quantified as Agatston score and mass score using the routinely used manufacturers' software with a default threshold for calcium scoring of 130 Hounsfield units (HU). The mass score calibration factor was calculated as described by McCollough et al. [16]. One observer performed the measurements with a semi-automatic method by selecting the calcification. For each individual calcification, median and range from the five measurements were calculated for both the Agatston score and mass score.

In order to interpret the results in terms of heart rate dependent CCS, the linear velocities of the artificial coronary arteries were converted to corresponding heart rates. Averaged over all coronary arteries at a cardiac cycle phase of $70 \%$ of the RR-interval, the average velocity of the coronary arteries during the acquisition phase is approximately 10,20 and $30 \mathrm{~mm} / \mathrm{s}$ for heart rates of $<60,60-75$ and $>75$ beats per minute (bpm), respectively [11].

\section{Statistical analysis}

Wilcoxon signed rank tests were used to compare Agatston and mass scores to the reference scores at $<60 \mathrm{bpm}$, and to compare mass scores to the physical mass. A p value smaller than 0.05 was used to indicate significant differences. Motion susceptibility was assessed with the cardiac motion susceptibility (CMS) Index, as described by Groen et al. [17]. This index gives a measure of the median deviation of the calcium scores over all heart rates from the CCS at rest. The CMS Index was calculated with the following equation:

$C M S=\frac{1}{N-1} \sqrt{\sum_{i=1}^{N}\left(x_{0}-x_{i}\right)^{2}} \frac{1}{x_{0}}$.

In this equation, $\mathrm{x}_{0}$ is the CCS at $0 \mathrm{bpm}$. The total number of heart rates is given by $\mathrm{N}$, and the CCS at heart rate $\mathrm{i}$ by $\mathrm{x}_{\mathrm{i}}$. A smaller CMS value signifies a lower motion susceptibility of CCS to cardiac motion. Kruskal Wallis tests were used to assess differences between calcium masses for each CT system and between CT systems, with a p value smaller than 0.05 indicating significant differences. All statistical analyses were performed with SPSS for Windows, version 22.0 (IBM corp., Armonk, NY, USA).

\section{Results}

CCS for the reference heart rate of $<60 \mathrm{bpm}$ are listed in Tables 2 and 3. At the reference heart rate, mass scores underestimated physical mass significantly for all 
calcification masses and all CT systems, except for the high $(p=0.500)$ and medium $(p=0.221)$ mass calcification for CT 2 and CT4 respectively. Example CT images for the high mass calcification at $>75 \mathrm{bpm}$ for all CT systems are shown in Fig. 4.

\section{Influence of heart rate on Agatston score}

The influence of increasing heart rates on Agatston scores for low, medium and high mass calcifications is shown in Table 2 and Fig. 5a. For the low mass calcification and all CT systems, Agatston scores at increasing heart rates were not significantly different from the reference Agatston score at $<60 \mathrm{bpm}$, regardless of the CT system. For the medium mass calcification at $>75 \mathrm{bpm}$, significant increases in Agatston scores were shown for all CT systems. Significant increases up to 50\% were also found for the high mass calcification. The influence of heart rate on the Agatston scores was different depending on the particular CT system. For example, at increased heart rate, CT3 showed an increase in Agatston score of $22 \%$ for the high mass calcification, while differences of $45-50 \%$ were found for the other CT systems.
Table 2 Reference Agatston scores at $<60 \mathrm{bpm}$ and percentage deviation from this reference as a function of heart rate for low mass $(38 \mathrm{mg})$, medium mass $(80 \mathrm{mg})$ and high mass $(157 \mathrm{mg})$ calcifications

\begin{tabular}{|c|c|c|c|c|c|c|}
\hline \multirow[t]{2}{*}{$\mathrm{CT}$} & \multirow[t]{2}{*}{ Calcification mass } & \multirow{2}{*}{$\begin{array}{l}\text { Reference } \\
\text { Median (range) }\end{array}$} & \multicolumn{2}{|l|}{$60-75 \mathrm{bpm}$} & \multicolumn{2}{|l|}{$>75 \mathrm{bpm}$} \\
\hline & & & Median \% (range) & $\mathrm{p}$ value & Median \% (range) & $\mathrm{p}$ value \\
\hline \multirow[t]{3}{*}{ CT1 } & Low & $99(95-136)$ & $-7(-31 ; 2)$ & 0.144 & $-21(-31 ; 6)$ & 0.078 \\
\hline & Medium & $351(344-375)$ & $4(-4 ; 30)$ & 0.176 & $32(11 ; 33)$ & $0.043 *$ \\
\hline & High & $515(450-553)$ & $-3(-11 ; 41)$ & 0.686 & $50(41 ; 58)$ & $0.043 *$ \\
\hline \multirow[t]{3}{*}{ CT2 } & Low & $105(79-120)$ & $-6(-21 ; 30)$ & 0.893 & $-9(-38 ; 11)$ & 0.279 \\
\hline & Medium & $315(267-347)$ & $11(1 ; 39)$ & $0.043^{*}$ & $33(7 ; 67)$ & $0.043 *$ \\
\hline & High & $421(387-459)$ & $14(2 ; 48)$ & $0.043^{*}$ & $49(19 ; 70)$ & $0.043^{*}$ \\
\hline \multirow[t]{3}{*}{ CT3 } & Low & 103 (89-117) & $-10(-20 ; 28)$ & 0.686 & $6(-18 ; 23)$ & 0.893 \\
\hline & Medium & $304(295-340)$ & $4(-2 ; 9)$ & 0.225 & $9(3 ; 19)$ & $0.043 *$ \\
\hline & High & $424(406-443)$ & $4(0 ; 12)$ & $0.043 *$ & $22(9 ; 28)$ & $0.042 *$ \\
\hline \multirow[t]{3}{*}{ CT4 } & Low & $108(105-132)$ & $-5(-17 ; 15)$ & 0.345 & $-10(-21 ; 1)$ & 0.080 \\
\hline & Medium & $367(320-377)$ & $33(23 ; 34)$ & $0.042 *$ & $26(14 ; 45)$ & $0.043 *$ \\
\hline & High & $505(472-544)$ & $38(36 ; 53)$ & $0.043 *$ & $45(44 ; 54)$ & $0.043 *$ \\
\hline
\end{tabular}

Results are indicated as median and total range values between brackets. Significant deviations from the reference Agatston scores are indicated by asterisks

Table 3 Reference Mass scores at $<60 \mathrm{bpm}$ and percentage deviation from this reference as a function of heart rate for low mass (38 mg), medium mass $(80 \mathrm{mg})$ and high mass $(157 \mathrm{mg})$ calcifications

\begin{tabular}{|c|c|c|c|c|c|c|c|}
\hline \multirow[t]{2}{*}{ CT } & \multirow[t]{2}{*}{ Calcifi-cation mass } & \multicolumn{2}{|l|}{ Reference } & \multicolumn{2}{|l|}{$60-75 \mathrm{bpm}$} & \multicolumn{2}{|l|}{$>75 \mathrm{bpm}$} \\
\hline & & Median (range) & $\mathrm{p}$ value & Median \% (range) & $\mathrm{p}$ value & Median $\%$ (range) & $\mathrm{p}$ value \\
\hline \multirow[t]{3}{*}{ CT1 } & Low & $31(28-35)$ & 0.078 & $-26(-33 ;-18)$ & $0.043 *$ & $-27(-33 ;-4)$ & $0.041^{*}$ \\
\hline & Medium & $76(74-78)$ & $0.043^{\dagger}$ & $-6(-21 ; 3)$ & 0.080 & $-9(-22 ; 1)$ & 0.078 \\
\hline & High & $145(127-153)$ & $0.043^{\dagger}$ & $-8(-25 ;-6)$ & $0.042 *$ & $-19(-21 ;-7)$ & $0.043^{*}$ \\
\hline \multirow[t]{3}{*}{$\mathrm{CT} 2$} & Low & $25(23-26)$ & 0.279 & $0(-12 ; 13)$ & 0.785 & $-13(-22 ;-4)$ & $0.043^{*}$ \\
\hline & Medium & $75(68-79)$ & $0.043^{\dagger}$ & $1(0 ; 10)$ & 0.102 & $8(0 ; 19)$ & 0.068 \\
\hline & High & $158(152-167)$ & $0.043^{\dagger}$ & $3(0 ; 15)$ & 0.068 & $15(7 ; 21)$ & $0.043^{*}$ \\
\hline \multirow[t]{3}{*}{ CT3 } & Low & $20(18-23)$ & 0.893 & $-11(-16 ; 5)$ & 0.221 & $-11(-13 ; 0)$ & 0.066 \\
\hline & Medium & $61(59-64)$ & $0.043^{\dagger}$ & $0(-2 ; 5)$ & 0.461 & $-2(-5 ; 8)$ & 0.892 \\
\hline & High & $128(127-132)$ & $0.042^{\dagger}$ & $1(0 ; 6)$ & 0.066 & $4(-2 ; 13)$ & 0.078 \\
\hline \multirow[t]{3}{*}{ CT4 } & Low & $28(26-30)$ & 0.080 & $-7(-18 ; 12)$ & 0.276 & $-18(-20 ;-12)$ & $0.042 *$ \\
\hline & Medium & $81(77-84)$ & $0.043^{\dagger}$ & $4(2 ; 12)$ & 0.042 & $1(-5 ; 5)$ & 0.683 \\
\hline & High & $170(167-175)$ & $0.043^{\dagger}$ & $12(6 ; 12)$ & $0.039^{*}$ & $9(4 ; 11)$ & $0.043^{*}$ \\
\hline
\end{tabular}

Results are indicated as median and total range values between brackets. Significantly different mass scores, in comparison with the physical mass, are indicated by an ${ }^{\dagger}$. Significant deviations from the reference mass scores are indicated by asterisks 


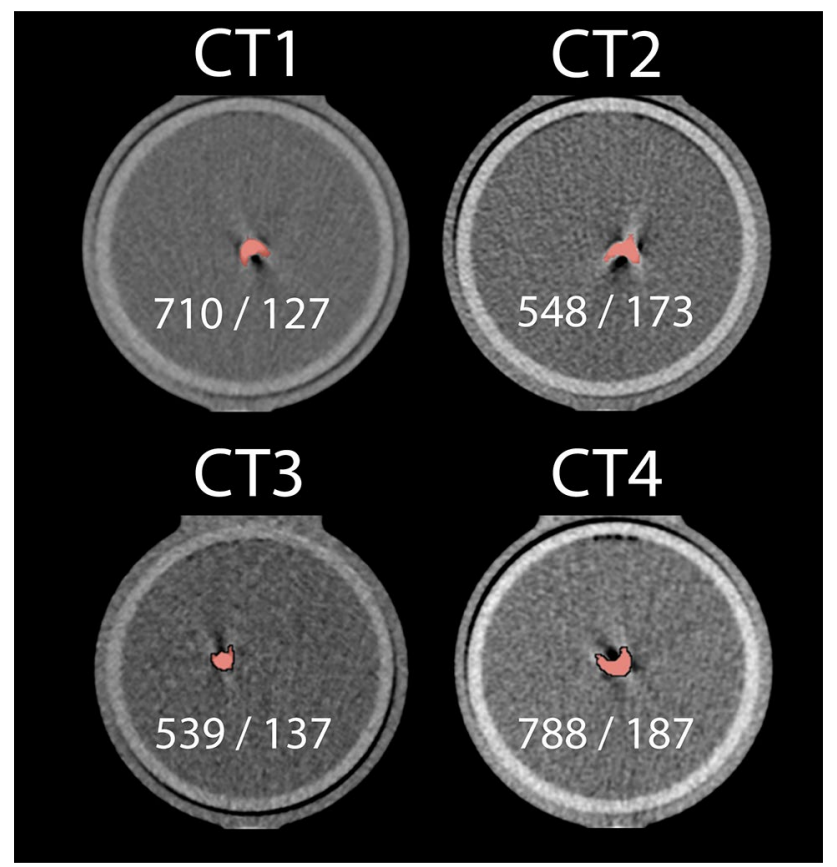

Fig. 4 Example images for all CT systems of the high mass (157 mg) calcification at $>75 \mathrm{bpm}$. Agatston/mass scores are given for each CT system

\section{Influence of heart rate on mass score}

Mass scores as a function of heart rate for the low, medium and high mass calcification are shown in Table 3. At 60-75 bpm, significantly different mass scores in comparison with the reference at $<60 \mathrm{bpm}$ were only shown for CT1 with the low mass calcification. At $>75 \mathrm{bpm}$, CT1 showed significantly different mass scores for the low mass and high mass calcification. The low mass and high mass calcification also showed significantly different mass scores for CT2. For CT3, all mass scores at $>75 \mathrm{bpm}$ were similar to the reference scores. CT4 showed significantly different mass scores for the low mass and high mass calcification.

In comparison with the physical mass, for all CT systems, mass scores for the low mass calcification were underestimated significantly $(\mathrm{p}<0.05)$ by $19-48 \%$ averaged over all heart rates (Fig. 5b). For the medium mass calcification at 60-75 bpm, CT4 showed an accurate approximation of the physical mass (deviation 7\%, $\mathrm{p}=0.080$ ), whereas the other CT systems showed significant underestimations $(\mathrm{p}<0.05)$ up to $23 \%$. At $>75 \mathrm{bpm}$, both CT2 and CT4 showed an accurate approximation of the physical mass (deviations $1 \%(\mathrm{p}=0.686)$ and $2 \%(\mathrm{p}=0.080)$, respectively $)$, whereas CT1 and CT3 underestimated the physical mass by $11 \%$ $(p=0.043)$ and $21 \%(p=0.043)$. For the high mass calcification, CT1 and CT3 underestimated the physical mass significantly $(\mathrm{p}<0.05$ ) up to $24 \%$ for $60-75$ and $>75 \mathrm{bpm}$. Significant overestimations were found for CT2 and CT4 at increased heart rates. At $60-75 \mathrm{bpm}$, mass scores deviated by $5 \%(p=0.043)$ and $20 \%(p=0.043)$ for CT2 and CT4 respectively, while differences of $15 \%(\mathrm{p}=0.043)$ and $19 \%$ $(\mathrm{p}=0.042)$ were found at $>75 \mathrm{bpm}$.

\section{CMS analysis}

Susceptibility of the Agatston and mass scores to motion, as expressed by CMS values, were smaller for mass scores, which indicates a smaller motion susceptibility of the mass score in comparison with the Agatston score (Fig. 6). Averaged over all calcification masses, the CMS value of CT3 was the smallest. Comparison of the CMS values of the three calcification masses per CT system showed significant differences between these values for CT1 $(p=0.009)$ and CT4 $(p=0.002)$. The motion susceptibility of the Agatston score for CT1 and CT4 therefore depends on the calcification density. For CT2 $(p=0.114)$ and CT3 $(p=0.054)$ CMS values of the three masses were not significantly different. Statistical analysis of the mass score CMS values showed similar results. Significant differences between CMS values of the different calcification masses were found for CT1 $(p=0.008)$ and CT4 $(\mathrm{p}=0.005)$, whereas CT2 $(\mathrm{p}=0.432)$ and CT3 $(p=0.050)$ did not show significant differences.

Differences in motion susceptibility between CT systems were significant. For the low mass calcification, susceptibility to motion as expressed in CMS values of the Agatston scores were comparable $(\mathrm{p}=0.564)$ between $\mathrm{CT}$ systems, whereas CMS values of the mass scores showed significant differences $(p=0.009)$. The medium mass calcification showed significantly different $(\mathrm{p}=0.043)$ Agatston score CMS values, while mass score CMS values were comparable $(p=0.687)$. For the high mass calcification, significant differences were shown for both the Agatston $(p=0.006)$ and mass score $(p=0.004)$ CMS values.

\section{Discussion}

This dynamic phantom study showed that for high-end CT systems of four major CT manufacturers coronary calcium scores were substantially, but not equally, influenced (up to $50 \%$ ) by heart rate. Motion susceptibility depends on both calcification mass and CT system. At increased heart rates, the stability of the mass score is superior to Agatston score stability.

The most important findings in this study were as follows. First, we found that no modern CT system is capable of completely mitigating the influence of heart rate on Agatston score or calcification mass. Second, CMS Index analysis showed significant differences for both Agatston and mass scores between four high-end CT systems. Also, different heart rates were found to differentially affect calcification 

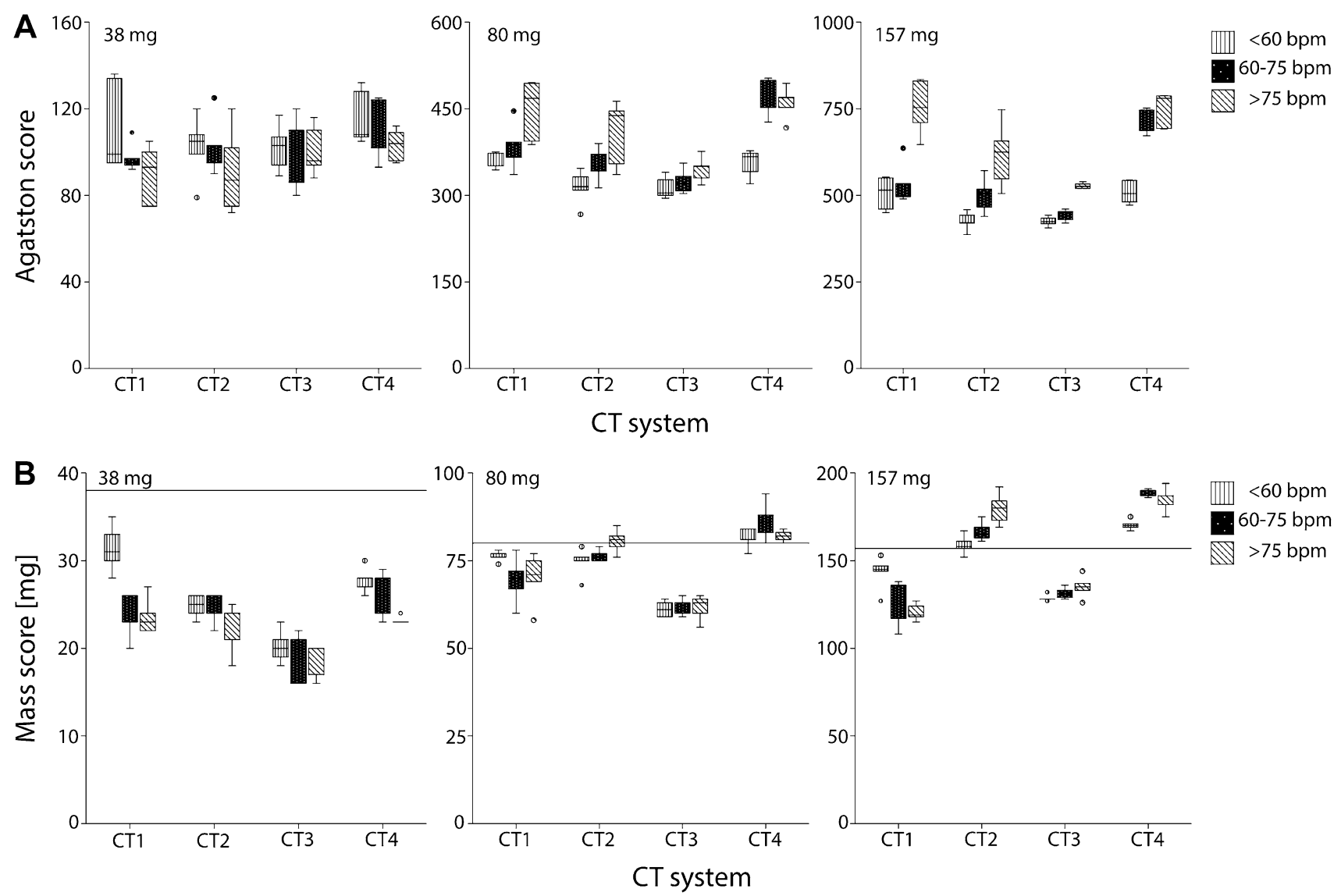

Fig. 5 a Agatston scores of the four artificial coronary arteries as found on CT-systems CT1-CT4 for calcification masses 38 (left),

coronary arteries as found on CT-systems CT1-CT4 for calcification 80 (middle) and $157 \mathrm{mg}$ (right). b Mass scores of the four artificial

masses 38 (left), 80 (middle) and $157 \mathrm{mg}$ (right). Physical mass of the calcification is represented with solid lines
Fig. 6 CMS Index for Agatston and mass scores for all CT systems and for the low (38 mg), medium $(80 \mathrm{mg})$ and high $(157 \mathrm{mg})$ mass calcification. Lower values indicate a smaller susceptibility to differences in motion, and therefore a smaller susceptibility to differences in heart rate

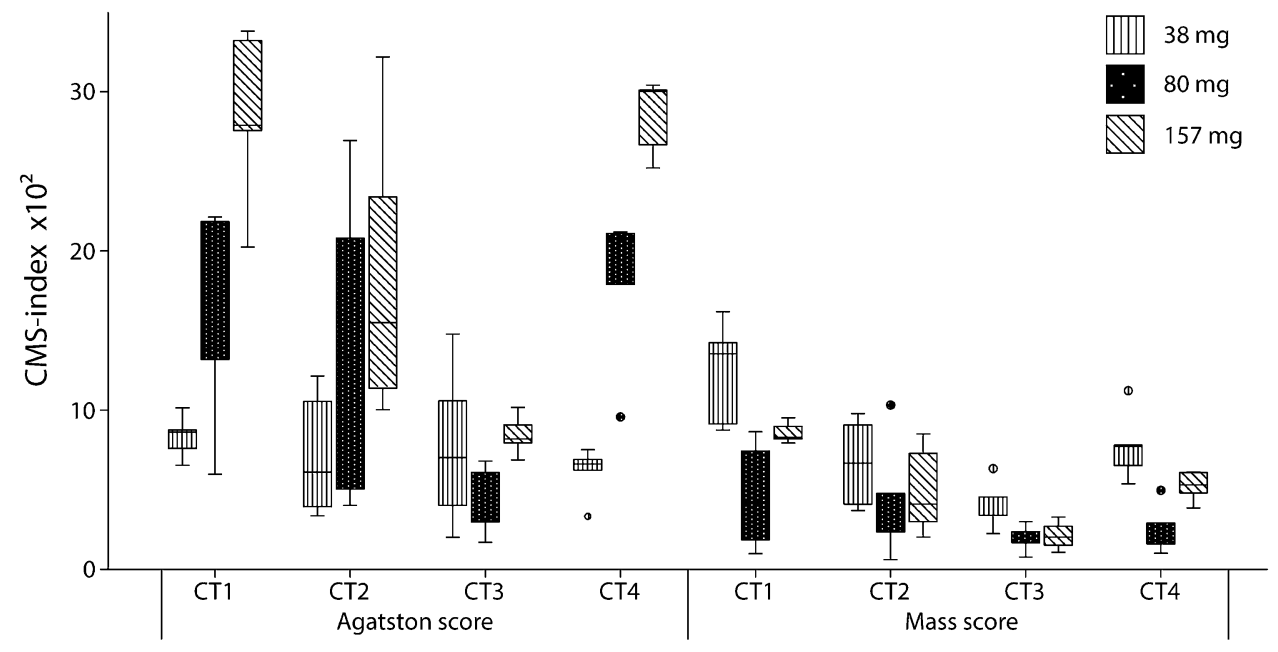

Calcium score - CT system masses for two CT systems. Thus, for these CT systems the influence of heart rate on CCS depends on calcification density.
For follow-up imaging a valid and precise scoring method is essential, therefore subsequent scans should be made with the same CT system and heart rates should be 
comparable. For low mass calcifications, voxels above the $130 \mathrm{HU}$ threshold at low heart rate might fall below this threshold as a result of motion blurring. As a result of this effect, CCS will decrease for these calcifications. For medium and high mass calcifications, the number of voxels above the $130 \mathrm{HU}$ threshold will increase as a result of motion blurring, thereby increasing CCS. The registered calcification size is smaller for CT systems with a higher temporal resolution, which is demonstrated in Fig. 4 for CT3 in comparison with the other CT systems. However, many other parameters, including acquisition and reconstruction parameters, also influence the registered calcification size [18]. In addition, technical advances, including dual-energy $\mathrm{CT}$ in combination with mono-energetic reconstructions, may influence the registered calcification quantification. With these mono-energetic reconstructions, it has been shown that blooming artefacts can be reduced [19]. Although especially for high density calcifications improved size registration is expected, the influence of these reconstructions on motion artefacts is unknown at this time. Third, the stability of the mass score is clearly superior to the stability of the Agatston score. This can be explained from the different way of calculating the mass score in comparison with the Agatston score. While the Agatston score considers the highest $\mathrm{HU}$ value in the lesion, the mass score can be thought of as a weighted average, which is therefore more robust to the effects of motion blurring. We found that the use of modern, stateof-the-art CT systems still results in substantial deviation from the true physical mass.

Our results are in line with a study conducted by Groen et al., who found decreased CCS for low mass calcifications and increased CCS for high mass calcifications at increased heart rates [7]. However, this study focused on only one CT system, while we used four high-end CT systems in the current study. Our findings are in agreement with the studies of Greuter et al. [6] and Groen et al. [7], who also showed the superior stability of mass scores in comparison to Agatston scores. These studies, however, were only performed on CT systems from one manufacturer. Tigges et al. used explanted coronary arteries mounted on a cardiac motion phantom to examine the influence of heart rate on CCS [8]. The explanted coronary arteries were subdivided into categories based on their respective Agatston score. The study showed a decrease in CCS with increasing heart rates, independent of calcification mass. For low mass calcifications these results correspond well to our results, whereas the high mass calcification results differ from our results. This difference in results probably originates from a difference in calcification density, because a high Agatston score can be the result of either a large calcification or a high density. The density of the calcification was not specified in the study of Tigges et al.
The current study has limitations. First, the artificial coronary arteries used in this study were translated at constant linear motion, whereas complex 3D motions are observed in-vivo. This 3D motion is patient and artery specific. Nevertheless, the scan times were relatively short as a result of fast rotation times, whereby the constant linear motion of our phantom was deemed sufficient as a model of the complex in vivo motion of coronary arteries. Second, linear velocity was converted to heart rates based on only one available study [11]. This limitation was accepted because of large variation in heart rates within the chosen heart rate groups $(<60,60-75$ and $>75 \mathrm{bpm})$. Third, slice thickness and increment were not the same for all CT systems. Previous studies demonstrated increased CCS for decreased slice thicknesses [20, 21]. However, the current study focusses on CCS from clinically used CCS protocols to evaluate the influence of heart rate within each CT system. Also, the deviating slice thickness and increment for CT1 in comparison with the other systems is in line with protocol recommendations as described previously by McCollough et al. [16]. With the clinically used CCS protocols, CCS were acquired with routine CT manufacturers' software. However, it was demonstrated by Weininger et al. that different types of CCS scoring software resulted in similar CCS [22].

In conclusion, the current dynamic phantom study showed that heart rate substantially influences CCS for the high-end CT systems of the four major CT manufacturers. Therefore, this study suggests that heart rate should be taken into account when interpreting the clinical impact of patients' calcium scores. Moreover, it is essential to acquire CCS scans on similar CT systems between follow-up studies, preferably at comparable heart rates. Also, mass scores were found to be more stable then Agatston scores.

\section{Compliance with ethical standards}

Conflict of interest The Radiology Department of the University Medical Center Utrecht received institutional grants from Philips Healthcare. Martin Willemink received personal fees for lectures from Philips Healthcare.

Research involving with human and animal participants This article does not contain any studies with human participants or animals performed by any of the authors.

Open Access This article is distributed under the terms of the Creative Commons Attribution 4.0 International License (http://creativecommons.org/licenses/by/4.0/), which permits unrestricted use, distribution, and reproduction in any medium, provided you give appropriate credit to the original author(s) and the source, provide a link to the Creative Commons license, and indicate if changes were made. 


\section{References}

1. Greenland P, Alpert JS, Beller GA et al (2010) 2010 ACCF/AHA guideline for assessment of cardiovascular risk in asymptomatic adults: executive summary: a report of the American College of cardiology foundation/American Heart association task force on practice guidelines. Circulation 122:2748-2764. https://doi. org/10.1161/CIR.0b013e3182051bab

2. Wilson PW, D'Agostino RB, Levy D et al (1998) Prediction of coronary heart disease using risk factor categories. Circulation 97:1837-1847. https://doi.org/10.1161/01.CIR.97.18.1837

3. Agatston AS, Janowitz WR, Hildner FJ et al (1990) Quantification of coronary artery calcium using ultrafast computed tomography. J Am Coll Cardiol 15:827-832

4. Goff DC, Lloyd-Jones DM, Bennett G et al (2014) 2013 ACC/ AHA guideline on the assessment of cardiovascular risk: a report of the American college of cardiology/American heart association task force on practice guidelines. Circulation 129:49-76. https:// doi.org/10.1161/01.cir.0000437741.48606.98

5. Bakhshi H, Ambale-Venkatesh B, Yang X et al (2017) Progression of coronary artery calcium and incident heart failure: the multiethnic study of atherosclerosis. J Am Heart Assoc 6:e005253. https://doi.org/10.1161/JAHA.116.005253

6. Greuter MJW, Groen JM, Nicolai LJ et al (2009) A model for quantitative correction of coronary calcium scores on multidetector, dual source, and electron beam computed tomography for influences of linear motion, calcification density, and temporal resolution: a cardiac phantom study. Med Phys 36:5079-5088. https://doi.org/10.1118/1.3213536

7. Groen JM, Greuter MJ, Schmidt B et al (2007) The influence of heart rate, slice thickness, and calcification density on calcium scores using 64-slice multidetector computed tomography: a systematic phantom study. Invest Radiol 42:848-855. https://doi. org/10.1097/RLI.0b013e318154c549

8. Tigges S, Arepalli CD, Tridandapani S et al (2012) A phantom study of the effect of heart rate, coronary artery displacement and vessel trajectory on coronary artery calcium score: potential for risk misclassification. J Cardiovasc Comput Tomogr 6:260-267. https://doi.org/10.1016/j.jcct.2012.01.005

9. Funabashi N, Irie R, Namihira Y et al (2015) Influence of tube voltage and heart rate on the Agatston calcium score using an in vitro, novel ECG-gated dual energy reconstruction 320 slice CT technique. Int J Cardiol 180:218-220. https://doi.org/10.1016/j. ijcard.2014.11.164

10. Achenbach S, Ropers D, Holle J et al (2000) In-plane coronary arterial motion velocity: measurement with electron-beam CT1. Radiology 216:457-463. https://doi.org/10.1148/radiology.216.2 r00au19457

11. Husmann L, Leschka S, Desbiolles L et al (2007) Coronary artery motion and cardiac phases: dependency on heart rate-implications for $\mathrm{CT}$ image reconstruction. Radiology 245:567-576. https://doi.org/10.1148/radiol.2451061791

12. Rutten A, Krul SPJ, Meijs MFL et al (2008) Variability of coronary calcium scores throughout the cardiac cycle: implications for the appropriate use of electrocardiogram-dose modulation with retrospectively gated computed tomography. Invest Radiol 43:187-194. https://doi.org/10.1097/RLI.0b013e31815cdd56

13. Hong C, Bae KT, Pilgram TK, Zhu F (2003) Coronary artery calcium quantification at multi-detector row CT: influence of heart rate and measurement methods on interacquisition variability initial experience. Radiology 228:95-100. https://doi.org/10.1148/ radiol.2281020685

14. Hong C, Bae KT, Pilgram TK (2003) Coronary artery calcium: accuracy and reproducibility of measurements with multi-detector row CT-assessment of effects of different thresholds and quantification methods. Radiology 227:795-801. https://doi. org/10.1148/radiol.2273020369

15. Willemink MJ, Vliegenthart R, Takx R P, et al (2014) Coronary artery calcification scoring with state-of-the-art CT scanners from different vendors has substantial effect on risk classification. Radiology 273:695-702. https://doi.org/10.1148/radiol.14140066

16. McCollough $\mathrm{CH}$, Ulzheimer S, Halliburton SS et al (2007) Coronary artery calcium: a multi-institutional, multimanufacturer international standard for quantification at cardiac CT. Radiology 243:527-538. https://doi.org/10.1148/radiol.2432050808

17. Groen JM, Greuter MJW, Vliegenthart R et al (2008) Calcium scoring using 64-slice MDCT, dual source CT and EBT: a comparative phantom study. Int J Cardiovasc Imaging 24:547-556. https://doi.org/10.1007/s10554-007-9282-0

18. van Ooijen PMA, Vliegenthart R, Witteman JCM, Oudkerk M (2005) Influence of scoring parameter settings on Agatston and volume scores for coronary calcification. Eur Radiol 15:102-110. https://doi.org/10.1007/s00330-004-2479-x

19. Hickethier T, Baeßler B, Kroeger JR et al (2017) Monoenergetic reconstructions for imaging of coronary artery stents using spectral detector CT: in-vitro experience and comparison to conventional images. J Cardiovasc Comput Tomogr 11:33-39. https:// doi.org/10.1016/j.jcct.2016.12.005

20. Muhlenbruch G, Thomas C, Wildberger JE et al (2005) Effect of varying slice thickness on coronary calcium scoring with multislice computed tomography in vitro and in vivo. Invest Radiol 40:695-699

21. Van Der Bijl N, De Bruin PW, Geleijns J et al (2010) Assessment of coronary artery calcium by using volumetric 320-row multidetector computed tomography: comparison of $0.5 \mathrm{~mm}$ with 3.0 mm slice reconstructions. Int J Cardiovasc Imaging 26:473-482. https://doi.org/10.1007/s10554-010-9581-8

22. Weininger M, Ritz KS, Schoepf UJ et al (2012) Interplatform reproducibility of CT coronary calcium scoring software. Radiology 265:70-77. https://doi.org/10.1148/radiol.12112532 